\title{
Crimen de agresión Crime of aggression
}

\author{
Gloria Fernández Arribas \\ Universidad Pablo de Olavide \\ gferarr@upo.es
}

Recibido / received: 23/02/2017

Aceptado / accepted: 31/07/2017

DOI: $\underline{\text { https://doi.org/10.20318/eunomia.2017.3824 }}$

\section{Resumen}

El crimen de agresión fue incluido como uno de los crímenes de competencia de la Corte Penal Internacional en el Estatuto de Roma, si bien en la actualidad la Corte no ejerce aún la competencia sobre el mismo. La definición del crimen de agresión, así como el régimen de entrada en vigor y ejercicio de la competencia, fue desarrollado en la Resolución 6 adoptada en la Conferencia de Kampala en $2010^{1}$, mediante la cual se enmienda el Estatuto de la Corte. En este trabajo se analiza, junto con la definición del crimen, el complicado y en ocasiones enrevesado régimen de entrada en vigor y ejercicio de la competencia, que difiere según los asuntos sean remitidos por un Estado miembro o proprio motu, o por el Consejo de Seguridad.

\section{Palabras clave}

Crimen de agresión, Corte Penal Internacional, entrada en vigor, jurisdicción, inmunidades.

\section{Abstract}

The crime of aggression was included in the Rome Statute as one of the crimes under the jurisdiction of the International Criminal Court, although the Court has not yet exercised its jurisdiction over this crime. The definition of the crime of aggression, as well as the regimes to entry into force and the exercise of jurisdiction were established in Resolution 6 adopted at the Kampala Conference in 2010, which amended the Rome Statute. This paper analyzes, together with the definition of crime, the complicated and sometimes intricate regime of entry into force and exercise of jurisdiction, which differs regarding whether the cases are referred by a Member State or proprio motu, or by the Security Council.

\section{Keywords}

Crime of aggression, International Criminal Court, entry into force, jurisdiction, immunities.

SUMARIO. 1. Introducción. 2. El crimen de agresión en el Estatuto de la Corte Penal Internacional. 2.1. Definición de crimen de agresión. 2.2. Entrada en vigor y ejercicio de la competencia. 2.2.1. Ejercicio de la competencia por remisión de un Estado o proprio motu. 2.2.2. Ejercicio de la competencia mediante remisión del Consejo de Seguridad. 2.3. Inmunidades.

\footnotetext{
${ }^{1}$ Conferencia de Revisión (2010), Resolución RC/Res.6 sobre el Crimen de Agresión.
} 


\section{Introducción}

La inclusión del crimen de agresión en el Estatuto de la Corte Penal Internacional (CPI) supuso un hito en la criminalización de lo que el Tribunal de Núremberg consideró como "el crimen internacional supremo, que difiere de los demás crímenes de guerra en que contiene en sí mismo el mal acumulado de todos ellos”2.

Pero a pesar de que el primer enjuiciamiento de carácter internacional por la comisión de crimen de agresión fue llevado a cabo por el Tribunal de Núremberg, previamente, con posterioridad a la Primera Guerra Mundial, ya se había intentado el enjuiciamiento del Kaiser Guillermo II; no por agresión, sino por "a supreme offence against international morality and the sanctity of treaties" (Tratado de Versalles, 1919, artículo 227). En este sentido, en la Conferencia de París de 1919 las potencias aliadas contestaron a las observaciones de Alemania relativas a las condiciones de paz señalando que la guerra que había dado comienzo el 1 de agosto de 1914 constituía el mayor crimen contra la humanidad y la libertad de los pueblos que cualquier nación que pudiera llamarse a sí misma civilizada había cometido jamás; y que por tanto "those individuals who are the most clearly responsable for German aggression and for those acts of barbarism and inhumanity which have disgraced the German conduct of the war, must be handed over to a justice" (ibid.). El resultado no fue en todo caso el esperado conforme a lo establecido en el Tratado de Paz, ya que el Káiser Guillermo II se refugió en los Países Bajos y nunca fue juzgado.

Será por tanto, como hemos señalado, en Núremberg donde por primera vez se produzca el enjuiciamiento por la comisión de crimen de agresión —definido en el Estatuto del Tribunal Militar Internacional como "Crímenes contra la paz", y señalando como tales "planificar, preparar, iniciar o librar guerras de agresión, o una guerra que constituya una violación de tratados, acuerdos o garantías internacionales, o participar en planes comunes o en una conspiración para lograr alguno de los objetivos anteriormente indicados" (Estatuto del Tribunal Militar Internacional de Nuremberg, 1945, artículo 6).

En todo caso, durante los juicios de Núremberg, tanto la Fiscalía como la Defensa plantearon dudas acerca de si el crimen de agresión era considerado un crimen con anterioridad al Estatuto de Núremberg, a lo que el Tribunal contestó que dicho crimen ya existía previamente en base al Pacto Briand-Kellogg de $1928^{3}$. Hoy día es ampliamente aceptado el crimen de agresión como un crimen de derecho internacional consuetudinario (Cryer, Friman, Robinson y Wilmshurst, 2014, p. 308).

\section{El crimen de agresión en el Estatuto de la Corte Penal Internacional}

Las propias características del crimen de agresión, junto con su conexión con el uso ilegal de la fuerza, hacen de este crimen una materia controvertida, lo cual ha llevado a establecer un régimen especial de entrada en vigor y ejercicio de la competencia por parte de la Corte.

El crimen de agresión fue incluido en el Estatuto de Roma entre los crímenes de competencia de la Corte, si bien no se incluía la definición del mismo ${ }^{4}$ —como sí

\footnotetext{
${ }^{2}$ Sentencia de 30 de septiembre/1 de octubre de 1946, citada en Remiro Brotons (2005, p. 1).

3 “... la solemne renuncia a la guerra como instrumento de política nacional necesariamente entraña la proposición de que una guerra de esa índole es ilegal en el derecho internacional, y de que quienes planean y hacen una guerra de esa índole, con sus inevitables y terribles consecuencias, están cometiendo un crimen al actuar de tal manera". Citado en PCNICC (2002).

${ }^{4}$ Sobre las posibles razones que llevaron a esta solución véase Cassese (1999, p. 146-148).
} 
ocurría con el resto de crímenes- y se señalaba que la Corte ejercería su competencia una vez se aprobara una disposición en la que se definiera el crimen así las condiciones en las que la Corte ejercería la mencionada competencia ${ }^{5}$.

Esta disposición se aprobó finalmente en el año 2010 en la Conferencia de Kampala (Conferencia de Revisión, 2010) en la cual se adopta la definición del crimen de agresión, las condiciones de ejercicio de la competencia y las enmiendas a los Elementos de los Crímenes (Asamblea de los Estados Partes en el Estatuto de Roma de la Corte Penal Internacional, 2002). A pesar de ello, la Corte no puede ejercer actualmente su competencia respecto del crimen de agresión, ya que conforme a los artículos 15 bis (3) y 15 ter (3), incorporados al Estatuto de Roma mediante al Resolución de Kampala, "la Corte ejercerá su competencia respecto del crimen de agresión de conformidad con el presente artículo, a condición de que se adopte una decisión después del $1^{\circ}$ de enero de 2017". Decisión que en la fecha de conclusión de este trabajo todavía no ha sido adoptada. Esta cuestión relativa a la entrada en vigor de las enmiendas y ejercicio de la competencia por parte de la Corte será analizada en un epígrafe aparte, junto con la definición del crimen de agresión y la aplicación de las inmunidades.

\subsection{Definición de crimen de agresión}

Tras previos intentos de definir el concepto de agresión (Cryer, Friman, Robinson y Wilmshurst, 2014, p. 309; Ferencz, 1972), finalmente la Asamblea General de Naciones Unidas adoptó en 1974 la Resolución 3314 en la que se incluía la definición de agresión, la cual posteriormente servirá de base para el concepto de crimen de agresión incluido en la Resolución de Kampala (Conferencia de Revisión, 2010), y hoy día en el artículo 8 bis del Estatuto de la Corte.

La definición recogida en el artículo 8 bis puede ser dividida en dos partes: la primera de ellas relativa a la determinación de la persona que comete un crimen de agresión, y la segunda referida a qué constituye un acto de agresión.

Respecto a la persona que comete un crimen de agresión, el artículo 8 bis (1) establece que será cometido por aquella persona que "estando en condiciones de controlar o dirigir efectivamente la acción política o militar de un Estado (...) planifica, prepara, inicia o realiza un acto de agresión que por sus características, gravedad y escala constituya una violación manifiesta de la Carta de Naciones Unidas".

La primera parte de esta definición indica que nos encontramos ante un crimen de liderazgo, esto es, un crimen que por norma general será cometido por jefes de Estado o Gobierno o altos cargos del Estado con control sobre la acción política o militar. Esta limitación deja fuera por tanto a los miembros de las fuerzas armadas que ejecutan el acto de agresión. Esta cuestión es además aclarada mediante la inserción del artículo 25 . 3 bis, que acota la aplicación de las reglas de la responsabilidad penal individual a las personas mencionadas, esto es, las que controlan o dirigen efectivamente la acción política o militar de un Estado.

En todo caso, y a pesar de que el crimen incluya la planificación, preparación e iniciación de un acto de agresión, los Elementos de los Crímenes (Asamblea de los Estados Partes en el Estatuto de Roma de la Corte Penal Internacional, 2002) indican que es necesario que el acto de agresión se haya llevado a cabo a fin de poder acusar a una persona de la comisión de dicho crimen. Pero no sólo esto, sino

\footnotetext{
${ }^{5}$ Artículo 5.2.
} 
que como en el resto de crímenes de competencia de la Corte, los Elementos de los Crímenes recogen un requisito relativo a la mens rea, de modo que la persona que comete el crimen tuviera conocimiento de que el uso de la fuerza armada utilizada era incompatible con la Carta de Naciones Unidas. Finalmente es necesario que dichos hechos constituyan también una violación manifiesta de la Carta.

Este último requisito -esto es, violación manifiesta de la Carta de Naciones Unidas- supone uno de los elementos más conflictivos de la definición del crimen de agresión, ya que a pesar de que en los Elementos de los Crímenes se señale que la expresión "manifiesta" es una calificación objetiva, los elementos del acto de agresión que permiten determinar dicho carácter manifiesto (características, gravedad y escala) sí podrían verse afectados por un cierto grado de subjetividad a la hora de llevar a cabo su análisis.

El análisis de este requisito debe ponerse por tanto en relación con la segunda parte de la definición: el acto de agresión.

El artículo 8 bis (2) toma la definición de agresión recogida en la Resolución 3314 y considera como acto de agresión "el uso de la fuerza armada por un Estado contra la soberanía, la integridad territorial o la independencia política de otro Estado, o en cualquier forma incompatible con la Carta de Naciones Unidas". A continuación, pasa a señalar una serie de actos que serán considerados como agresión y que ya recogía también la Resolución 3314. En todo caso es necesario señalar que no nos encontramos ante una lista cerrada, y por tanto cualquier otro acto que cumpla los requisitos señalados podrá ser considerado también acto de agresión.

En relación con los requisitos que debe cumplir el acto de agresión, la necesidad de que se trate de una violación manifiesta de la Carta ha sido señalado como un medio para dar cumplimiento al requisito de que el artículo 8 bis sea interpretado de conformidad con el derecho consuetudinario en la materia (Kreß, 2016, pp. 509-510), limitando de este modo los actos de agresión que dan lugar a responsabilidad del individuo a aquellos que son prohibidos por el derecho consuetudinario. En este sentido, se ha señalado que el establecimiento de este umbral parece querer dejar a un lado aquellas actuaciones que implican el uso de la fuerza armada y que hoy día resultan controvertidas (Cryer, Friman, Robinson y Wilmshurst, 2014, p. 231; Heinsch, 2010, p. 730; Kreß, 2010, p. 1138), como serían la intervención humanitaria, el uso de la fuerza en la lucha contra el terrorismo o determinados casos de legítima defensa, hechos que podrían ser suficientemente graves y, en escala, importantes como para cumplir los demás requisitos, pero sobre los que los que no existe posición unánime acerca de su contravención con la Carta de Naciones Unidas. Aquí cumpliría su función la expresión "características", estableciendo como señala Kreß un umbral cualitativo (2016, pp. 1138).

En todo caso, la gravedad y escala también permiten acotar el campo de actuación respecto de otros casos de uso de la fuerza que, si bien constituyen una violación de los principios de la Carta por su gravedad o escala, no parece que entrasen en el concepto de violación manifiesta, como por ejemplo serían las escaramuzas fronterizas; se requiere por tanto intensidad (Kreß, 2016, pp. 511). La Corte Internacional de Justicia (en adelante $\mathrm{CIJ}$ ) ya señaló que las formas más graves de uso de la fuerza que daban lugar al ejercicio de la legitima defensa eran las que implicaban un ataque armado, y la propia definición de agresión recoge expresamente el uso de la fuerza armada; pero la $\mathrm{ClJ}$, al excluir los incidentes fronterizos de entre las formas más graves del uso de la fuerza, señalaba también que era necesaria cierta intensidad (Corte Internacional de Justicia, 1986, par. 195). 
El acto de agresión recogido en el estatuto de la CPI cumple por tanto con esa necesidad de intensidad que ha venido desarrollando la $\mathrm{ClJ}^{6}$, y que se encuentra en conexión con el derecho consuetudinario relativo al uso de la fuerza. Este requisito de intensidad también puede considerase confirmado en el Entendimiento número 6 , que establece que "se entiende que la agresión es la forma más grave y peligrosa del uso ilegal de la fuerza" (Kreß, 2016, p. 514). La gravedad del acto también fue señalada en la Resolución 3314, al establecer que el Consejo de Seguridad podría concluir que el uso de la fuerza armada por parte de un Estado podía no constituir un acto de agresión debido a que el hecho o sus consecuencias no eran los suficientemente graves.

\subsection{Entrada en vigor y ejercicio de la competencia}

El régimen de entrada en vigor de las enmiendas adoptadas en la Conferencia de Kampala sobre el crimen de agresión viene recogido en el artículo 121.5 del Estatuto de la $\mathrm{CPI}$, tal y como recoge la propia Resolución 6 (Conferencia de Revisión, 2010). De este modo, las enmiendas que recogen la definición de crimen de agresión y ejercicio de la competencia entrarán en vigor sólo para los Estados partes que las hayan adoptado, y un año después del depósito de los instrumentos de ratificación. El artículo 121.5 excluye en todo caso la competencia de la Corte respecto de los crímenes cometidos en el territorio o por nacionales de Estados partes que no hayan aceptado las enmiendas. Esta última frase, junto con el contenido del artículo 1 de la Resolución 6, relativo a la posibilidad de que un Estado parte deposite una declaración mediante la cual no reconozca la competencia de la Corte en los casos de remisión por un Estado o proprio motu, ha dado lugar a diversas interpretaciones, y supone uno de los diferentes aspectos propios del complicado y enrevesado régimen de entrada en vigor y ejercicio de la competencia respecto de este crimen ${ }^{7}$.

En lo referido al ejercicio de la competencia es necesario referirse, en primer lugar, al retraso en la activación de la misma, ya que a pesar de que las enmiendas entren en vigor en el momento de ratificación o aceptación por parte del Estado, la Corte, tal y como señalan los artículos 15 bis (3) y 15 ter (3), deberá esperar a que después de 1 de enero de 2017 la mayoría de Estados partes que se requieren para la aprobación de una enmienda - esto es, dos tercios- adopten una decisión respecto del ejercicio de la competencia.

\subsubsection{Ejercicio de la competencia por remisión de un Estado o proprio motu}

En Kampala se decidió establecer dos regímenes distintos para el ejercicio de la competencia según la remisión fuese realizada por un Estado y proprio motu, o por el Consejo de Seguridad.

Respecto de la remisión por un Estado o proprio motu, el artículo 15 bis (2) establece la primera diferencia, ya que a pesar de que el artículo 121.5 que mencionábamos no exigía un número mínimo de ratificaciones para la entrada en vigor de las enmiendas, el artículo 15 bis (2) establece que la competencia podrá ejercerse sólo un año después de la ratificación o aceptación de las enmiendas por treinta Estados partes; requisito ya cumplido, y exigencia ésta recogida en el artículo 121.4, que establece un régimen distinto de entrada en vigor. Por tanto, parece ser

\footnotetext{
${ }^{6}$ Véase Corte Internacional de Justicia (2003, par. 43 ss.).

${ }^{7}$ Sobre los problemas que plantea el régimen de entrada en vigor y ejercicio de la competencia véase (Akande 2010), Kress y Von Holtzendorff (2010), Zimmermann (2012), Fernández Arribas (2013), Barriga y Blokker (2016).
} 
que se ha optado por tomar el modelo de entrada en vigor del artículo 121.4 a fin de limitar el ejercicio de la competencia de la Corte.

El siguiente límite en el ejercicio de la competencia lo encontramos en los artículos 15 bis (4) y (5), referidos a los casos en los que un Estado parte haya depositado una declaración señalando que no acepta la competencia de la Corte en relación con los crímenes de agresión resultantes de actos de agresión cometidos por dicho Estado. Esta declaración, conocida también como cláusula opting out, podría considerase superflua ya que el Estado puede optar por no ratificar las enmiendas y de ese modo, conforme al artículo 121.5, quedaría fuera de la competencia de la Corte. Pero tal y como se ha apuntado, es posible que el Estado, a pesar de querer quedar fuera de la competencia de la Corte, desee llevar a cabo la ratificación de las enmiendas a fin de que pueda cumplirse con el requisito de ratificación de treinta Estados partes señalado, y de ese modo que la Corte pueda ejercer su competencia respecto de otros Estados, así como que pueda ejercerla en los casos de remisión por el Consejo de Seguridad, tal y como veremos en el siguiente epígrafe. Y por otra parte, esta declaración no excluiría, como sí lo hace el artículo 121.5, el ejercicio de la competencia en caso de que el crimen de agresión hubiese sido cometido en el territorio de dicho Estado, ya que el artículo 121.5 excluye el crimen cometido por nacionales o en territorio del Estado; respecto de este último la doctrina entiende como territorio tanto aquel donde " el crimen es trazado, como en aquel sobre el que tiene sus efectos, esto es, tanto en territorio del Estado agresor como del Estado víctima" (Fernández Arribas, 2011, p. 467) ${ }^{8}$.

En relación con los Estados no partes, el mencionado artículo 15 bis (5) establece una nueva excepción respecto del artículo 12.2, ya que excluye de la competencia de la Corte los crímenes de agresión cometidos por nacionales o en el territorio de Estados no partes.

Por otra parte, el papel del Consejo de Seguridad en la determinación de la existencia de un crimen de agresión como requisito previo para que la Corte pudiera ejercer su competencia fue discutido durante la Conferencia de Kampala ${ }^{9}$, y sus efectos y el resultado se observan en el artículo 15 bis (6), (7) y (8). En este sentido, y a pesar de cumplirse los requisitos señalados anteriormente, el Fiscal, cuando decida iniciar la investigación, deberá verificar si el Consejo de Seguridad se ha pronunciado sobre la existencia de un acto de agresión, además de notificar al Secretario General de Naciones Unidas la situación ante la Corte. El Fiscal en esta situación puede encontrarse con que el Consejo de Seguridad sí haya confirmado la existencia de un acto de agresión, lo que le permitiría iniciar la investigación; o puede encontrarse con el caso más probable ${ }^{10}$ de que el Consejo de Seguridad no haya constatado expresamente la existencia de un acto de agresión. En este último caso deberá esperar seis meses desde que se notificó al Secretario General sobre la situación, y en caso de transcurrido ese periodo de tiempo sin pronunciamiento por parte del Consejo de Seguridad, el Fiscal podrá iniciar la investigación previa

\footnotetext{
${ }^{8}$ Barriga y Blokker (2016, p. 637) señalan por otra parte que la cláusula opting out lleva a la conclusión de que la jurisdicción de la Corte se ejerce, por defecto y se si cumplen el resto de requisitos, respecto de actos de agresión cometidos por cualquier Estado parte, incluso respecto de aquellos que no han ratificado las enmiendas. Entendemos que este caso la Corte podría iniciar una investigación respecto de acto de agresión cometido por un Estado parte, pero solo podría ejercer su competencia respecto del crimen en el caso de que el autor o el territorio donde se cometa el crimen perteneciesen a un Estado que hubiese ratificado las enmiendas, en caso contrario nos encontraríamos con una contradicción entre dicha cláusula opting out y el artículo 121.5 del Estatuto.

${ }^{9}$ Véase Van Schaack (2010, p. 513).

10 Tal y como señala Remiros ro tons "en la práctica, sin embargo, el Consejo de Seguridad ha sido reacio a calificar como agresión situaciones que aparentaban serlo, prefiriendo cultivar la ambig edad" (2005, p. 3).
} 
autorización por la Sala de Cuestiones Preliminares, y siempre que el Consejo de Seguridad no se haya pronunciado en contra conforme al artículo 16 del Estatuto.

En relación con la determinación de un acto de agresión por parte del Consejo de Seguridad, el artículo 15 bis no señala que el Consejo deba realizar dicha determinación de manera expresa. Esto nos lleva a preguntarnos si sería suficiente que el Consejo de Seguridad determinara que se ha producido un acto de los señalados anteriormente como actos de agresión sin que expresamente lo calificara como tal. De este modo cuando el Consejo de Seguridad condenaba expresamente en su resolución 660 (1990) la invasión de Kuwait por Irak —acto incluido en el artículo 8 bis (2) a) como acto de agresión, añadiendo que dicho acto constituía un quebrantamiento de la paz y la seguridad internacionales, lo que permitiría confirmar la gravedad del mismo-, estaría, conforme a la interpretación propuesta, determinando la existencia de un acto de agresión que permitiría el ejercicio de la competencia por remisión del Fiscal conforme al artículo 15 bis (6).

Finalmente señalar que la determinación de la existencia de un acto de agresión por parte del Consejo de Seguridad u otro órgano ajeno a la Corte no comprometerá las conclusiones a las que ésta pueda llegar.

\subsubsection{Ejercicio de la competencia mediante remisión del Consejo de Seguridad}

Las exigencias relativas al número de ratificaciones, así como a la adopción de una resolución después de 1 de enero de 2017, se mantienen también para el ejercicio de la competencia por remisión del Consejo de Seguridad.

Pero a diferencia de las remisiones por los Estados o proprio motu, en el caso del Consejo de Seguridad no se establecen excepciones, de modo que el Consejo de Seguridad podrá remitir un asunto relativo a actos cometidos en territorio y por nacionales de Estados no partes. Este hecho, si bien supone una importante diferencia a la hora de luchar contra la impunidad por la comisión de este crimen, se encuentra con el complicado problema que presenta el reconocimiento de inmunidades a los jefes de Estado y de Gobierno, así como a otros altos representantes del Estado, y que analizaremos brevemente en el siguiente epígrafe.

En todo caso, este reconocimiento de competencia respecto de Estados no partes podría plantear dudas respecto de los Estados partes que no han ratificado las enmiendas. Conforme al artículo 121.5 del Estatuto, las enmiendas entrarán en vigor respecto de aquellos Estados que las hayan ratificado, lo que conllevaría en principio que, en caso de que un Estado no ratificase las enmiendas, no le sería de aplicación el artículo 15 ter relativo a las remisiones por el Consejo de Seguridad. Esta interpretación del artículo 121.5 no resultaría coherente con el contenido de la Resolución 6 y en concreto del artículo 15 ter, ya que mientras que el Consejo de Seguridad sí podría remitir asuntos sobre Estados no partes en el Estatuto de Roma, no podría remitirlos respecto de Estados partes que no hayan ratificado las enmiendas. Esta cuestión queda en todo caso solventada en el Anexo II de la Resolución 6, en cuyo apartado 2 se recoge que la Corte ejercerá la competencia en casos de remisión por el Consejo de Seguridad "independientemente de que el Estado de que se trate haya aceptado la competencia de la Corte a este respecto".

Esta declaración resulta también acorde con la exclusión de la cláusula opting out del artículo 15 ter; de este modo, los Estados, al ratificar las enmiendas, no podrán declarar que no aceptan la competencia de la Corte respecto de los casos remitidos por el Consejo de Seguridad. 


\subsection{Inmunidades}

Finalmente señalaremos brevemente el problema planteado respecto del derecho consuetudinario relativo a las inmunidades de jefes de Estado y de Gobierno, así como de otros altos cargos, junto con el hecho de que nos encontremos ante un crimen de liderazgo que sólo pueda ser cometido precisamente por aquellos a los que se les reconocen dichas inmunidades.

En primer lugar, es necesario señalar que, respecto de los Estados partes, dicho problema en principio no debería plantearse en el Estatuto de la Corte, ya que el artículo 27 del Estatuto establece que "las inmunidades y las normas de procedimiento especiales que conlleve el cargo oficial de una persona, con arreglo al derecho interno o al derecho internacional, no obstarán para que la Corte ejerza su competencia sobre ella". De este modo los Estados partes estarían renunciando a las inmunidades que corresponden a los cargos oficiales.

El problema, por otra parte, se plantea respecto de los Estados no partes en el Estatuto que no han renunciado a las inmunidades de los altos cargos conforme al artículo 27. A este hecho se une el contenido del artículo 98 del Estatuto, que establece que la Corte no dará curso a una solicitud de entrega en los casos en los que la misma imponga al Estado requerido actuar de forma incompatible con las obligaciones que le imponga el derecho internacional respecto de la inmunidad de un Estado, inmunidad diplomática de una persona o de un tercer Estado.

La conexión entre el artículo 27 y 98 ha sido objeto de numerosos estudios y ha tenido su manifestación práctica en el caso Al Bashir (Abrisketa Uriarte, 2016; Fernández Arribas, 2015), en el que la Corte se ha enfrentado a la falta de colaboración de algunos Estados partes alegando en determinados casos el reconocimiento de la inmunidad a Al Bashir como jefe de Estado.

En todo caso la cuestión de la inmunidad de los altos cargos adquiere un grado de especial importancia en el caso del crimen de agresión. Como hemos señalado, nos encontramos ante un crimen de liderazgo, por lo que sólo podrá ser cometido por aquellas personas a las que se les reconoce inmunidad de jurisdicción. Si bien los Estados partes renuncian a dicha inmunidad conforme al artículo 27, el hecho de que se reconozca la inmunidad a los altos cargos de los Estados no partes en la práctica viene a dejar sin aplicación, en parte, el artículo 15 ter, que permite al Consejo de Seguridad remitir asuntos a la Corte con independencia de que el crimen haya sido cometido en el territorio o por un nacional de un Estado no parte. Ello se debe a que en dichos casos y en aplicación estricta del artículo 98, la Corte únicamente podría ejercer su jurisdicción en caso de que el Estado del nacional requerido renunciara a dicha inmunidad, caso poco probable al ser una decisión que principalmente correspondería a la persona cuya entrega se está solicitando.

Por tanto, mediante dicha aplicación del artículo 98, nos encontraríamos en la práctica con los mismos efectos del artículo 15 bis (5), que establecía que "respecto de un Estado no parte en el presente Estatuto, la Corte no ejercerá su competencia respecto del crimen de agresión cuando éste sea cometido por los nacionales de ese Estado ...", apartado este que no se añadió al artículo 15 ter con la intención de otorgar competencia a la Corte respecto de los nacionales de Estados no partes en casos de remisión de asuntos por el Consejo de Seguridad.

Será por tanto necesario buscar interpretaciones que permitan dar cumplimiento al objeto y fin de este artículo 15 ter, de modo que la competencia que se ha pretendido otorgar a la Corte respecto de Estados no partes no quede 
desvirtuada y sin efecto por la aplicación del régimen de inmunidades, y ello a pesar de, y teniendo presentes que, tal y como indicaba la CIJ, "immunity from criminal jurisdiction and individual criminal responsibility are quite separate concepts. While jurisdictional immunity is procedural in nature, criminal responsibility is a question of substantive law" (Corte Internacional de Justicia, 2002, par. 60).

Bibliografía

ABRISKETA URIARTE, J. (2016), "Al ashir: ¿Excepción a la inmunidad del jefe de Estado de Sudán y cooperación con la Corte Penal Internacional?, Revista española de Derecho Internacional, vol. 68.

AKANDE, D. (2010), "What Exactly was Agreed in Kampala on the Crime of Aggression?", EJIL: Talk!. Disponible en: http://www.ejiltalk.org/what-exactlywas-agreed-in-kampala-on-the-crime-of-aggression/

BARRIGA, S. y BLOKKER, N. (2016), "Entry into force and conditions for the exercise of jurisdiction: cross-cutting issues". En: KREß, C. y BARRIGA, S., The Crime of Aggression. A Commentary, Cambridge University Press, Cambridge.

CASSESE, A. (1999), "The Statute of the International Criminal Court: Some Preliminary Reflections", European Journal of International Law, vol. 10.

CRYER, R., FRIMAN, H., ROBINSON, D. y WILMSHURST, E. (2014), An Introduction to International Criminal Law and Procedure, Cambridge University Press, Cambridge.

FERENCZ, B.B. (1972), "Defining Aggression: Where it Stands and Where it's going", The American Journal of International Law, vol. 66.

FERNÁNDEZ ARRIBAS, G. (2011), "El Compromiso de Kampala sobre el Crimen de Agresión. ¿Fin a la impunidad por el uso ilegal de la fuerza?”. En: RITO, W. y PUEYO LOSA, J. (dirs.), Conflitos Armados, Gestão Pós- Conflitual e Reconstrução. Conflictos Armados, Gestión Posconflicto y Reconstrucción, Andavira.

FERNÁNDEZ ARRIBAS, G. (2013), "Entrada en vigor y ejercicio por la Corte Penal Internacional de la jurisdicción sobre el crimen de agresión", Revista Electrónica de Estudios Internacionales, núm. 25.

FERNÁNDEZ ARRIBAS, G. (2015), "Las dificultades de la Corte Penal Internacional para el enjuiciamiento de Jefes de Estado y de Gobierno: los casos Al Bashir y Kenyatta", Revista Española de Derecho Internacional, Información y Documentación, vol. 67.

HEINSCH, R. (2010), "The Crime of Aggression After Kampala: Success or Burden for the Future?", The Goettingen Journal of International Law, vol. 2, núm. 2.

KREß, C. (2010), "Time for Decision: Some thoughts on the Immediate Future of the Crime of Aggression: A reply to Andreas Paulus", The European Journal of International Law, vol. 20, núm. 4.

KREß, C. (2016), "The state conduct element". En: KREß, C. y BARRIGA, S., The Crime of Aggression. A Commentary, Cambridge University Press, Cambridge.

KRESS, C. y VON HOLTZENDORFF, L. (2010), "The Kampala Compromise on the Crime of Aggression", Journal of International Criminal Justice, núm. 8.

REMIRO BROTÓNS, A. (2005), "Agresión, crimen de agresión, crimen sin castigo", FRIDE, Documento de Trabajo, 10.

ZIMMERMANN, A. (2012), "Amending the Amendment Provisions of the Rome Statute. The Kampala Compromise on the Crime of Aggression and the Law of Treaties", Journal of International Criminal Justice, no 10.

VAN SCHAACK, B. (2010), "Negotiating at the Interface of Power \& Law: The Crime of Aggression", Columbia Journal of Transitional Law, vol. 49.

Documentos oficiales 
ASAMBLEA DE LOS ESTADOS PARTES EN EL ESTATUTO DE ROMA DE LA CORTE PENAL INTERNACIONAL (2002), "Elementos de los Crímenes", Primer período de sesiones, Nueva York, 3 a 10 de septiembre de 2002, Documentos Oficiales, pp. 112-160. Disponible en:

http://www.ordenjuridico.gob.mx/Jurlnt/CP5.pdf

ASAMBLEA GENERAL DE NACIONES UNIDAS (1974), Resolución 3314 (XXI) de 14 de diciembre.

CARTA DE LAS NACIONES UNIDAS (1945)

CONFERENCIA DE REVISIÓN (2010), Resolución RC/Res.6 sobre el Crimen de Agresión. Disponible en: https://crimeofaggression.info/documents/6/RC-Res6SPA.pdf

CORTE INTERNACIONAL DE JUSTICIA (1986), "Actividades militares y paramilitares en y contra Nicaragua (Nicaragua c. Estados Unidos de América)", sentencia del 27 de junio, Recueil 1986.

CORTE INTERNACIONAL DE JUSTICIA (2002), Orden de arresto de 11 de Abril de 2000 (Repúblic Democrática del Congo c. Bélgica), sentencia del 14 de febrero, Recueil, 2002.

CORTE INTERNACIONAL DE JUSTICIA (2003), "Plataformas petrolíferas (República Islámica de Irán c. Estados Unidos de América)", sentencia del 6 de noviembre, Recueil 2003.

ESTATUTO DEL TRIBUNAL MILITAR INTERNACIONAL DE NUREMBERG (1945).

NACIONES UNIDAS, A/CONF. $183 / 9$ (1998), Estatuto de Roma de la Corte Penal Internacional

PCNICC (COMISIÓN PREPARATORIA DE LA CORTE PENAL INTERNACIONAL) (2002), "Examen histórico de la evolución en materia de agresión (PCNICC/2002NGCA/L.1)", Grupo de Trabajo sobre el crimen de agresión, Nueva York.

TRATADO DE VERSALLES (1919). Disponible en:

http://fama2.us.es/fde/ocr/2006/tratadoDeVersalles.pdf

TRIBUNAL MILITAR INTERNACIONAL DE NUREMBERG (1946), Sentencia de 30 de septiembre/1 de octubre, American Journal of International Law (1947), vol. 4. 